\title{
Representativeness of EN 1040/13727 Assay Conditions for Evaluating In Vitro the Bactericidal Activity of a Chlorhexidine Digluconate and Benzalkonium Chloride Antiseptic Preparation
}

\author{
S. Salvatico1, C. Feuillolay1, V. Jabbour², C. Gouhier-Kodas², C. Roques ${ }^{3,4}$ \\ ${ }^{1}$ Fonderephar, Faculté des Sciences Pharmaceutiques, Toulouse, France \\ ${ }^{2}$ Laboratoire Innotech International, Arcueil, France \\ ${ }^{3}$ Laboratoire de Génie Chimique UMR 5503, Faculté des Sciences Pharmaceutiques, Toulouse, France \\ ${ }^{4}$ Service de Bactériologie-Hygiène, CHU Toulouse, Hôpital Purpan, Toulouse, France \\ Email: ch.roques@wanadoo.fr
}

How to cite this paper: Salvatico, S., Feuillolay, C., Jabbour, V., Gouhier-Kodas, C. and Roques, C. (2018) Representativeness of EN 1040/13727 Assay Conditions for Evaluating In Vitro the Bactericidal Activity of a Chlorhexidine Digluconate and Benzalkonium Chloride Antiseptic Preparation. Open Journal of Medical Microbiology, 8, 56-73.

https://doi.org/10.4236/ojmm.2018.83006

Received: July 31, 2018

Accepted: September 8, 2018

Published: September 11, 2018

Copyright ( 2018 by authors and Scientific Research Publishing Inc. This work is licensed under the Creative Commons Attribution International License (CC BY 4.0).

http://creativecommons.org/licenses/by/4.0/

\begin{abstract}
Aims: The representativeness of the mandatory bacterial strains specified in European standards for in vitro assay of the bactericidal activity of antiseptics was evaluated by testing the activity of an antiseptic combining chlorhexidine digluconate $0.2 \%$ and benzalkonium chloride $0.5 \%$ against 21 additional bacterial strains, and the positive interaction between these two biocidal agents was assessed. Methods and Results: The bactericidal activity of the antiseptic solution used pure or diluted was assessed according to the European standards EN 1040 and EN 13727. The contact time was $1 \mathrm{~min}$ at $20^{\circ} \mathrm{C}$. Interfering substances used in the EN 13727 assay were bovine serum albumin and sheep erythrocytes, simulating "dirty" conditions, and hard water. A reduction of colony-forming units by $\geq 5 \log _{10}$ was deemed to meet the requirements to conclude bactericidal activity. Under "basic" conditions, the bactericidal activity of the antiseptic was observed against all four mandatory strains specified in the standards as well as against nearly all the additional strains tested, including most of those with acquired antibiotic-resistance. The positive interaction between the two biocidal agents was also confirmed. Under "dirty" conditions, the bactericidal activity of the antiseptic solution was maintained against all the mandatory strains and was reduced against only four of the additional strains tested. Conclusions: With regard to the antiseptic tested and under the experimental conditions described, bactericidal activity evidenced against the mandatory strains appeared to be representative of
\end{abstract}


that manifested against a wide range of the main pathogenic bacteria. Reduced bacterial activity against some of the additional strains tested (e.g. Enterobacteriaceae) was observed under "dirty" conditions. Significance and Impact of the Study: EN 13727 with some experimental adjustments represents an additional appropriate standard that needs to be considered for mucocutaneous antiseptic assessment. However, it may be worth including other specific bacterial strains to those specified in the standard, when evaluating antiseptics intended for use in certain clinical situations.

\section{Keywords}

Antiseptic, In Vitro Bactericidal Activity, Chlorhexidine, Benzalkonium Chloride, Interfering Substances, EN 1040, EN 13727

\section{Introduction}

Over the past decade, antiseptics have been increasingly used for prophylactic or therapeutic purposes, notably as a result of the continuing rise in the frequency of antibiotic resistance in the healthcare setting [1]. When used appropriately, antiseptics can decrease cutaneous or mucosal microbial colonization by reducing both transient and commensal flora [2], and their clinical benefit in controlling infection is well established.

Many antiseptic formulations including active substances such as chlorhexidine, iodine or chlorine derivatives, or quaternary ammonium compounds (QACs), are currently available. The choice of an antiseptic and the optimal conditions of its use should be guided by its known antimicrobial spectrum, the targeted situations or indications (e.g. pre-procedural antisepsis, antiseptic treatment of skin, wounds or mucous membranes) and the potential risks of adverse effects related to each ingredient and to the formulation as a whole. Some mucocutaneous antiseptic agents, such as chlorhexidine or iodine derivatives, may have a broad spectrum of activity against the main bacteria, whereas others, like QACs, may kill a smaller range of disease-causing organisms depending on their membrane structure (Gram-positive or Gram-negative) [3].

The spectrum of bactericidal activity of antiseptics is primarily determined using in vitro tests carried out according to internationally accepted standards and generally consisting in determination of the reduction in the number of viable test microorganisms by pure or diluted solutions of the antiseptic. The methodology used in this study conforms to that described in European (EN) standards. The standard EN 14885 [4] describes the application of European standards to chemical disinfectants and antiseptics and more specifically indicates the recommended and/or mandatory assays for active substances and newly marketed products: basic in vitro bactericidal activity (phase 1), in vitro activity under the conditions of intended use (phase 2, step 1), in vivo activity after standardized bacterial inoculation on skin (phase 2 step 2) and in vivo activity 
under conditions of practical use in humans (phase 3).

The standard EN 1040 [5] implemented in April 2006 and never updated since then, describes the phase 1 assay recommended to test the basic bactericidal activity of an active antiseptic substance or a formulated product under development. This assay may also be used, for instance, to assess potential interaction(s) between active substances or excipients. Bactericidal activity is determined against Pseudomonas aeruginosa ATCC 15442 and Staphylococcus aureus ATCC 6538 , after a 5 -minute contact time at $20^{\circ} \mathrm{C}$, in the absence of any organic contaminant.

The standard EN 13727 [6] is a phase 2 step 1 standard, describing the assay required for the in vitro bactericidal assessment of final, market-ready formulations of hand hygiene products. The aim of this assay is to establish the bactericidal activity of the product under simulated practical conditions relevant to its intended use (e.g. at dilutions and time points corresponding to the actual patterns of use of the final antiseptic preparation).

Although phase 2 standards for assay of the bactericidal activity of hygienic and surgical hand washes and hand rubs are well described in EN 14885 [4], there is currently no phase 2 standard that specifically addresses evaluation of the bactericidal activity of mucocutaneous antiseptics in other simulated conditions of use. Yet the presence of many organic (proteins) or inorganic (electrolytes, divalent cations) substances found in biological fluids, such as blood, exudates or pus, decreases or even completely inhibits the in vitro bactericidal activities of numerous marketed antiseptic solutions and individual active substances [2] [7] [8] [9]. These decreases in bactericidal activity have also been observed in vivo [7] [10] [11] [12] [13] [14].

The assay described in the standard EN 13727 [6] has already been used to assess the bactericidal activity of mucocutaneous antiseptics in the presence of interfering substances, i.e. under conditions resembling as closely as possible those pertaining to their routine use in medical practice [15].

Two kinds of interfering substances are specified in this standard: a mixture of bovine albumin and erythrocytes for "dirty" conditions, or bovine albumin alone for "clean" conditions. The current version of EN 13727 [6] requires the use of four different test organisms: two Gram-positive cocci: Staphylococcus aureus ATCC 6538 and Enterococcus hirae ATCC 10541, and two Gram-negative bacilli: Escherichia coli K12 NCTC 10538 and Pseudomonas aeruginosa ATCC 15442 and stipulates specific contact times for hygienic or surgical hand washes and hand rubs.

The strains specified in EN 13727 [6] and in EN 1040 [5] are deemed to be representative of the most clinically relevant bacteria found in health care settings, in terms of spectrum and pathophysiological involvement [16] [17]. Hence, E. coli, P. aeruginosa, $S$. aureus and Enterococci are the leading pathogens responsible for the majority of nosocomial infections [17] [18]. However, the bacteriology of the commonly isolated (nosocomial) pathogens has changed 
over time. For example, Proteus spp., Klebsiella spp. and Escherichia spp. were responsible for nosocomial infections in the 1960s, while recent surveys have indicated that streptococci, along with coagulase-negative and coagulase-positive staphylococci, have re-emerged, whereas the incidence of $K$. pneumoniae and $E$. coli has declined [17]. Consequently, with the development of new emergent and sometimes challenging nosocomial pathogens and in the currently evolving context of bacterial resistance to antimicrobials, it is worth questioning whether the bactericidal activity of an antiseptic formulation against the four mandatory strains stipulated in European standards may truly predict their effectiveness against normal flora and cutaneous pathogens. Although corresponding to the species responsible for the majority of nosocomial infections, these reference strains therefore represent only a subset of the bacteria of concern. It is worth noting that in the USA, time-kill tests, based on a principle similar to that of the European suspension tests, must be performed against 23 laboratory strains considered to be representative of nosocomial pathogens [19].

With the aim of investigating the representativeness of the assay conditions imposed by European standards, we tested a marketed antiseptic solution combining chlorhexidine digluconate (CHX) $0.2 \%$ and benzalkonium chloride (BZK) $0.5 \%$ (Dermobacter ${ }^{\circ}$, Laboratoire Innotech International) previously confirmed to be bactericidal according to the standard NF EN 13727 [15] and commonly used for injured skin and superficial wound antisepsis. Our objectives were to address the following questions:

1) Is the bactericidal activity of this tested antiseptic preserved under "dirty" conditions and after a short contact time against both the mandatory and other relevant bacterial strains?

2) Do assays employing the two test strains stipulated in EN 1040 [5] and the four test strains specified in EN 13727 [6] provide sufficient evidence of the comprehensive bactericidal activity of this antiseptic?

We therefore investigated the in vitro spectrum of antibacterial activity against the mandatory test strains stipulated in the European standards EN 1040 [5] and EN 13727 [6] as well as against 21 other pathogens of concern in health care settings including antibiotic-resistant strains (such as methicillin-resistant Staphylococcus species or vancomycin-resistant Enterococcus species) and other bacteria with pathogenic potential for the skin or mucosa. Finally, the known positive interaction between the two active substances included in this antiseptic preparation was checked.

\section{Materials and Methods}

\subsection{Solutions}

The antiseptic solutions tested are described below:

- BZK 0.5\% (w/v) solution (Laboratoire Innotech International, batch DER-1303-007), 
- CHX 0.2\% (w/v) solution (Laboratoire Innotech International, batch DER-1303-008),

- CHX 0.2\% + BZK 0.5\% solution (Laboratoire Innotech International, batch DER-1303-009),

- Dermobacter ${ }^{\circledast}$, solution for cutaneous application combining CHX 0.2\% and BZK 0.5\% (Laboratoire Innotech International, batch CH106). This licensed antiseptic is a foaming solution indicated for the cleansing and adjuvant treatment of skin and mucous membrane disorders, either primarily of bacterial origin or susceptible to bacterial superinfection. In practice, the solution is used pure on skin and diluted 1:10 on mucous membranes, intensive rinsing being required following the application. Excipients comprise coco alkyl dimethyl betaines, ethylene oxide and propylene oxide copolymers, hydroxyethylcellulose, monohydrated citric acid and sodium citrate.

\subsection{Bacterial Strains}

The bacterial strains stipulated by the standards EN 1040 [5] and/or EN 13727 [6] for bactericidal assay were used, namely $S$. aureus CIP 4.83 (ATCC 6538), $P$. aeruginosa CIP 103467 (ATCC 15442), E. coli CIP 54117 (NCTC 10538) and E. hirae CIP 58.55 (ATCC 10541). These strains were provided by the Pasteur Institute Collection (Paris, France). Receipt strains were treated as recommended by Pasteur Institute Collection and then stored at $-80^{\circ} \mathrm{C}$ in a glycerol-based cryoprotective solution to be in compliance with the standard NF EN 12353. A further 21 strains were also tested, giving a total of 25 bacterial strains. These additional strains included both referenced strains (from the Pasteur Institute Collection [CPI]; or the American Type Culture Collection [ATCC]) and clinical isolates (from the University Hospital, Toulouse, France), corresponding to the species most commonly involved in cutaneous and/or mucosal infections. The distribution between Gram-positive and Gram-negative bacteria was as follows:

- Gram-positive strains:

Aerobes and aerotolerants: Staphylococcus epidermidis CIP 6821, Streptococcus pyogenes CIP 5641T, Streptococcus agalactiae CIP 106884, Corynebacterium amycolatum CIP $403452 \mathrm{~T}$ and strains expressing acquired resistance: Staphylococcus aureus MRSA ATCC 33591, Staphylococcus aureus VISA CIP 106757 and Enterococcus faecium VanA CIP 107387;

Anaerobes/microaerophiles/capnophiles including: Propionibacterium acnes CIP 53117T, Clostridium perfringens CIP 103409, Finegoldia magna CIP 103666, Gardnerella vaginalis CIP 7074T.

- Gram-negative strains:

Aerobes and aerotolerants: Klebsiella pneumoniae CIP 8291, Enterobacter cloacae CIP 6058T, Serratia marcescens CIP 103716, Proteus mirabilis CIP 103181T, Citrobacter freundii CIP 57.32T, Acinetobacter baumanii CIP 70.34T and strains expressing acquired resistance: ESBL-producing Klebsiella pneumoniae CTXM15 (clinical isolate), Stenotrophomonas maltophilia tono R (clinical isolate); 
Anaerobes/microaerophiles/capnophiles: Bacteroides fragilis AIP 7716, Neisseria gonorrhoeae CIP 7918T.

Cultures were maintained and colony-forming units (CFU) were counted on: Tryptone soy agar (Biomérieux, Crapone, France; casein peptone $15.0 \mathrm{~g}$, soy peptone $5.0 \mathrm{~g}$, sodium chloride $(\mathrm{NaCl}) 5.0 \mathrm{~g}$, agar $15.0 \mathrm{~g}$, and water to $1 \mathrm{~L}$ ) for the main aerobes and facultative anaerobes; Columbia agar (Biomérieux, Crapone, France; meat and casein peptone $10.0 \mathrm{~g}$, hydrolyzed animal protein $10.0 \mathrm{~g}$, heart peptone $3.0 \mathrm{~g}$, corn starch $1.0 \mathrm{~g}, \mathrm{NaCl} 5.0 \mathrm{~g}$, agar $13.5 \mathrm{~g}$, and water to $1 \mathrm{~L}$ ) for Corynebacterium amycolatum CIP 403452T (after the addition of 10\% neutralizer for CFU counts); Columbia agar + 5\% sheep erythrocytes (Biomérieux; Crapone, France; meat and casein peptone $10.0 \mathrm{~g}$, hydrolyzed animal protein $10.0 \mathrm{~g}$, heart peptone $3.0 \mathrm{~g}$, corn starch $1.0 \mathrm{~g}, \mathrm{NaCl} 5.0 \mathrm{~g}$, agar $13.5 \mathrm{~g}$, sheep blood $15 \mathrm{ml}$, and water to $1 \mathrm{~L}$ ) for Gardnerella vaginalis CIP 7074T, under anaerobic conditions for Propionibacterium acnes CIP 53117T, Clostridium perfringens CIP 103409, Finegoldia magna CIP 103666 and Bacteroides fragilis AIP 7716; Chocolate PolyViteXagar (Biomérieux, Crapone, France; casein peptone $7.5 \mathrm{~g}$, meat peptone $7.5 \mathrm{~g}$, corn starch $1.0 \mathrm{~g}$, dipotassium phosphate $4.0 \mathrm{~g}, \mathrm{NaCl} 5.0 \mathrm{~g}$, hemoglobin bovine $10.0 \mathrm{~g}$, agar $10.0 \mathrm{~g}$, PolyViteX $10 \mathrm{~mL}$, and water to $1 \mathrm{~L}$ ) under $5 \% \mathrm{CO}_{2}$ for Neisseria gonorrhoeae CIP 7918T; and Tryptone soy agar under $5 \% \mathrm{CO}_{2}$ for Streptococcus agalactiae CIP 106884 and under aerobiosis for the other strains with the addition of $10 \%$ neutralizer for Streptococcus pyogenes CIP $5641 \mathrm{~T}$ (incubation $36^{\circ} \mathrm{C} \pm 1^{\circ} \mathrm{C}-24$ to $48 \mathrm{~h}$ ).

\subsection{Trial Protocols}

1) Evaluation of the Bactericidal Spectrum and Sensitivity to Interfering Substances

Bactericidal activity against $S$. aureus CIP 4.83, P. aeruginosa CIP 103467, and 23 additional strains was tested according to protocol described in EN 1040 (Table 1) [5]. Bacterial suspensions were prepared in tryptone salt at concentrations ranging from $1.5 \times 10^{8}$ to $5.0 \times 10^{8} \mathrm{CFU} \mathrm{mL}^{-1}$, with final test concentrations ranging between $1.5 \times 10^{7}$ and $5.0 \times 10^{7} \mathrm{CFU} \mathrm{mL} \mathrm{m}^{-1}$. The tested antiseptic was diluted in water for injectable preparations (to concentrations of $80 \%, 50 \%$ and $10 \% \mathrm{v} / \mathrm{v}$ ). Preliminary assays were performed to define the range of dilutions allowing discrimination of differences in antibacterial activity. The tests were conducted according to standard conditions ( $5 \mathrm{~min} \pm 10 \mathrm{~s}$ contact duration at a temperature of $20^{\circ} \mathrm{C} \pm 1^{\circ} \mathrm{C}$ ) and for an additional contact time of $1 \mathrm{~min} \pm 5$ $\mathrm{s}$, to allow comparison with the results obtained using the protocol described in EN 13727 [6].

The experimental conditions specified in EN 13727 [6] for hygienic and surgical hand rubs and washes were if necessary adapted according to the conditions of use of the tested antiseptic in its various indications. In particular, certain indications require dilution of the antiseptic prior to use. Notably, for use specifically on the vaginal mucosa (against Neisseria gonorrhoeae, Gardnerella vaginalis) and on wounds (against Clostridium perfringens), the product 
Table 1. Assay conditions adapted from the European standards EN 1040 and EN 13727.

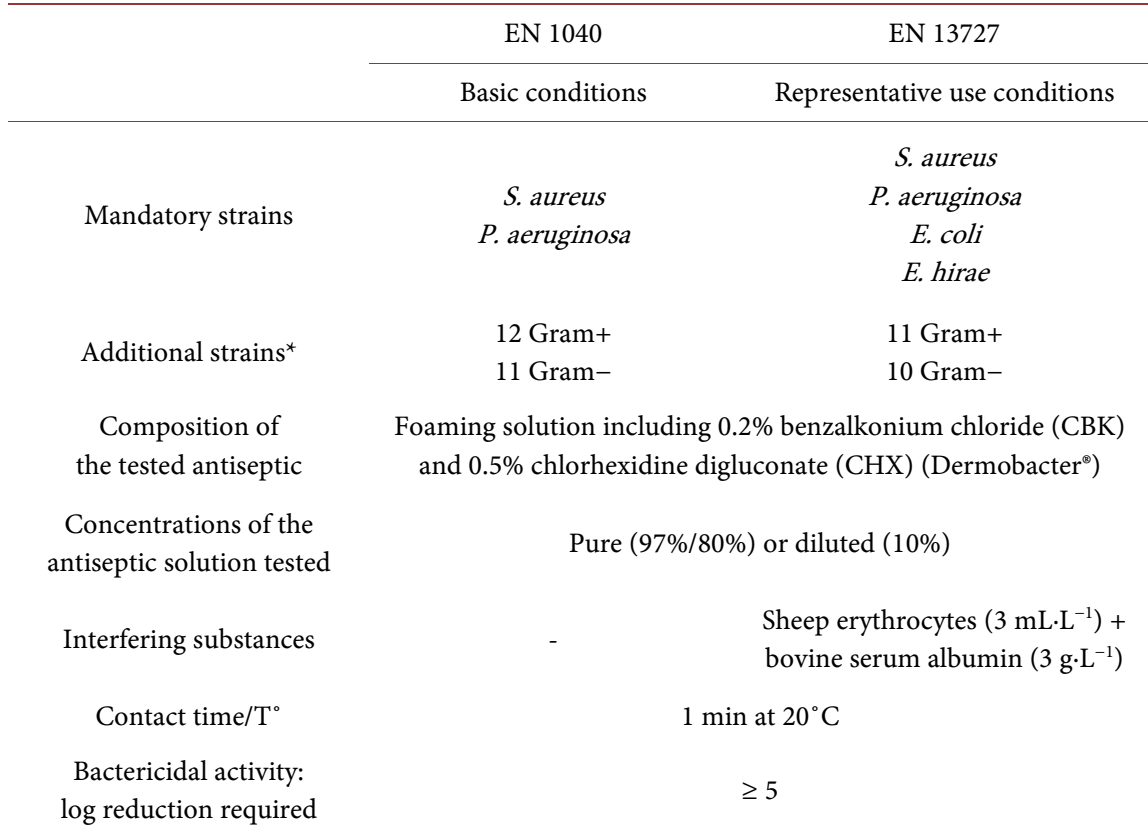

*Strains commonly involved in mucocutaneous infections including some with acquired resistance to antibiotics (MRSA, ESBL-producing Klebsiella pneumoniae, VRE). ESBL: extended-spectrum $\beta$-lactamase; MRSA: methicillin-resistant Staphylococcus aureus; VRE: vancomycin-resistant Enterococcus species. Two vaginal strains tested only after dilution according to EN 13727.

is always diluted. The highest recommended concentrations of $97 \%$ or $80 \%(\mathrm{v} / \mathrm{v})$ were employed for the ready-to-use product, $50 \%$ or $10 \%(\mathrm{v} / \mathrm{v})$ concentrations being employed to reflect use of the diluted product. The theoretically inactive concentration tested was $1 \%$ for the four mandatory bacterial strains stipulated in the European standard and $0.1 \%$ for the additional strains.

The most restrictive assay conditions were selected, i.e. dilutions in hard water (recommended for the application of diluted solutions) and contact under "dirty conditions" (recommended to simulate antiseptic use without previous cleaning of the application zone). Hard water is a saline solution including magnesium chloride, calcium chloride, sodium bicarbonate, that simulates tap water, with a hardness of $30^{\circ} \mathrm{F}$ (equivalent to 21 English degrees). Divalent cations play a considerable role in antiseptic activity and are therefore considered as potentially interfering substances [2] [7] [8] [9]. For "dirty conditions", the interfering substances comprised a mixture of $3 \mathrm{~g} \cdot \mathrm{L}^{-1}$ bovine serum albumin (Sigma Aldrich, Saint-Quentin Fallavier, France) and $3 \mathrm{~mL} \cdot \mathrm{L}^{-1}$ of sheep erythrocytes (Biomérieux, Crapone, France).

Assays were performed at a temperature of $20^{\circ} \mathrm{C} \pm 1^{\circ} \mathrm{C}$ and with a contact duration of $1 \mathrm{~min} \pm 5 \mathrm{~s}$, more restrictive than the maximum contact time of $5 \mathrm{~min}$ stipulated in the standard for surgical hand rubs and washes.

The absence of any toxic effect of the experimental conditions and the neutralizer (or filtration procedure), as well as the validation of the dilution neutralization step was checked as specified in the standards EN 1040 and EN 13727 [5] 
[6]. The neutralizer used in the assays comprised Tween 80 (10\%), saponin (2\%), lecithin (2\%), sodium thiosulfate (0.5\%) (Sigma Aldrich), and Trypcase soy broth (Biomérieux). Filtration rather than neutralization was used for assays of bactericidal activity against $G$. vaginalis (nitrocellulose membrane $0.45 \mu \mathrm{m}-$ Millipore-rinsing solution: sterile distilled water $2 \times 100 \mathrm{~mL}$ ), and $N$. gonorrhoeae (Durapore hydrophobe membrane $0.45 \mu \mathrm{m}-$ Millipore-rinsing solution: sterile distilled water $2 \times 100 \mathrm{~mL}$ ).

After neutralization or filtration, residual viable bacteria (CFU) were counted after incubation in the appropriate agar for the control and the test suspensions. The CFU counts recorded at this time, expressed in decimal logarithms $\left(\log _{10}\right.$ values) were subtracted from the basal values determined prior to antiseptic exposure in order to calculate the log reductions in CFU count. The upper limit of the CFU count indicating bactericidal activity, as defined by the standard, corresponds to a lower limit in terms of log reduction (expressed as $<3$ in the results tables). The lower limit of the CFU count defined by the standard induces an upper limit in terms of log reduction ( $\geq 5$ in the results tables). The tested antiseptic solution is considered bactericidal according to EN 1040 [5] at concentrations reducing the initial bacterial count by $\geq 5$ log. According to EN 13727 [6], the tested antiseptic solution is considered bactericidal for hygienic and surgical hand rubs if, when used undiluted, it produces a $\geq 5 \log$ reduction in CFU counts; and for hygienic hand washes or for surgical hand washes if, when used diluted ( $\leq 50 \%$ ), it produces a $\geq 5 \log$ or $\geq 3 \log$ reduction respectively. In this study, bactericidal activity against specific microorganisms was considered to be evidenced when a $\geq 5 \log$ reduction in CFU counts was observed in assays performed according to EN 1040 requirements and based on the most restrictive acceptance criteria for hand hygienic friction (NF EN 13727) [5] [6].

2) Assessment of CHX-BZK Interaction

The test conditions stipulated in EN 1040 [5] were applied to confirm the positive interactions between CHX and BZK activities when combined such as in the tested licensed antiseptic. The bactericidal activities of CHX $0.2 \%(w / v)$ and BZK 0.5\% (w/v) separately, of the combination CHX - BZK, and of the licensed antiseptic Dermobacter ${ }^{\oplus}$ were tested against Staphylococcus aureus CIP 4.83 and Pseudomonas aeruginosa CIP 103467 at the concentrations and ratio present in Dermobacter ${ }^{\oplus}$ and the results compared. Bacterial suspensions and dilutions of each antiseptic preparation were made as described above. Assays were conducted according to the standard, at a temperature of $20^{\circ} \mathrm{C} \pm 1{ }^{\circ} \mathrm{C}$ and with a contact time of $5 \mathrm{~min} \pm 10 \mathrm{~s}$.

\section{Results}

\subsection{Spectrum of Bactericidal Activity and Sensitivity to Interfering Substances}

As shown in Table 2, the bactericidal activity of Dermobacter ${ }^{\circledR}$ observed after

1 minute of contact and in the absence of any interfering substance (EN 1040 
Table 2. Bactericidal activity of Dermobacter ${ }^{\circledR}$ under basic conditions according to the standard EN 1040 (contact time: $1 \mathrm{~min}$ ).

\begin{tabular}{|c|c|c|c|}
\hline & \multicolumn{3}{|c|}{ Concentration $(\mathrm{v} / \mathrm{v})$} \\
\hline & $80 \%$ & $50 \%$ & $10 \%$ \\
\hline \multicolumn{4}{|l|}{ Mandatory strains } \\
\hline \multicolumn{4}{|l|}{ Staphylococcus aureus CIP 4.83} \\
\hline Pseudomonas aeruginosa CIP 103467 & & & \\
\hline
\end{tabular}

Additional Gram + strains

Enterococcus hirae CIP 58.55

Staphylococcus epidermidis CIP 6821

Streptococcus pyogenes CIP $5641 \mathrm{~T}$

Streptococcus agalactiae CIP 106884

Corynebacterium amycolatum CIP 103452T

Propionibacterium acnes CIP 53117T

Clostridium perfringens CIP 103409

Finegoldia magna CIP 103666

Gardnerella vaginalis CIP 7074T

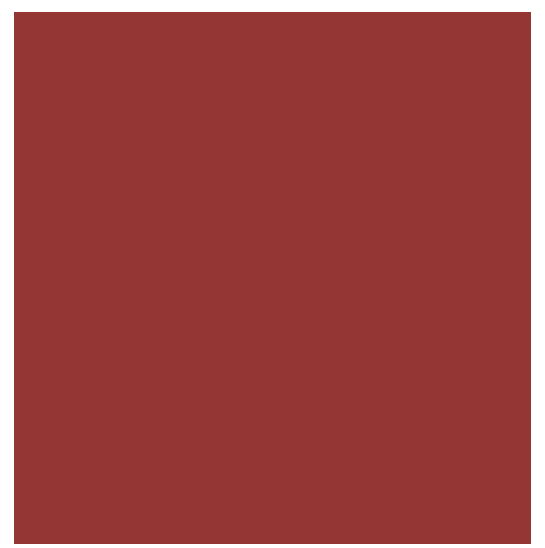

Additional Gram + strains with acquired antibiotic resistance

Staphylococcus aureus MRSA ATCC 33591

Staphylococcus aureus VISA CIP 106757

Enterococcus faecium VAN A CIP 107387

Additional Gram—strains

Escherichia coli CIP 54.117

Klebsiella pneumoniae IP 8291

Enterobacter cloacae CIP 6058T

Serratia marcescens CIP 103716

Acinetobacter baumannii CIP 70.34T

Citrobacter freundii CIP 57.32T

Proteus mirabilis CIP 103181T

Bacteroides fragilis AIP 7716

Neisseria gonorrhoeae CIP 7918T

Additional Gram-strains with acquired antibiotic resistance

ESBL-producing Klebsiella pneumoniae CTXM15

Stenotrophomonas maltophilia tonoR
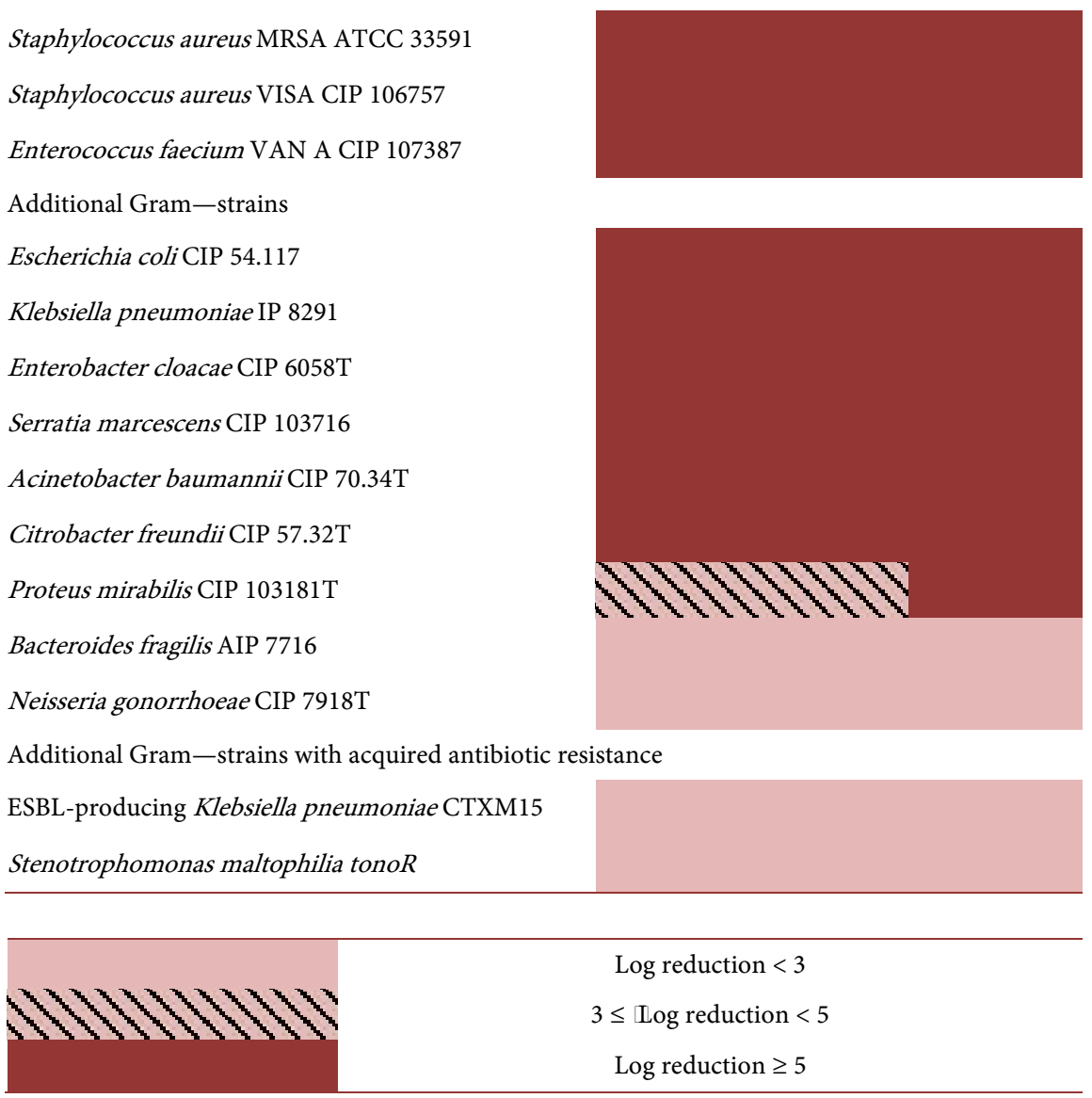

Log reduction $<3$

$3 \leq$ Log reduction $<5$

Log reduction $\geq 5$

The theoretically inactive concentration tested was $0.1 \%$ (data not shown). Bactericidal activity is expressed as a logarithmic reduction at the tested concentrations $80 \%, 50 \%$ and $10 \%$. 
protocol, [5]) was demonstrated against both Gram-positive and Gram-negative bacteria, including anaerobes and microaerophiles and, above all, against strains expressing acquired antibiotic resistance (log reduction in CFU count $\geq 5$ ). Under these experimental conditions, the bactericidal activity of the antiseptic was sustained even when diluted to $10 \%(\mathrm{v} / \mathrm{v})$. The only concentration bactericidal against Proteus mirabilis was $10 \%(\mathrm{v} / \mathrm{v})$, the log reduction in CFU count noted for this strain at the higher concentrations being under 5 (4.59 at $80 \%$ with a contact time of $5 \mathrm{~min}$, and 4.53 at $50 \%$ with a contact time of $1 \mathrm{~min}$ ).

Under drastic conditions, including dilution of the product in hard water and "dirty" conditions (as specified in EN 13727 [6]), the bactericidal activity of Dermobacter ${ }^{\oplus}$ was for the most part preserved, even after a short $(1 \mathrm{~min})$ contact time (Table 3 ). At the $10 \%$ concentration, the antiseptic achieved a log reduction in CFU count $\geq 5$ on 22 out of 25 strains and a log reduction $\geq 3$ but $<5$ on 23 out of 25 strains. Its bactericidal activity was nevertheless reduced under these conditions against C. perfringens CIP 103409 (50\%: $4.29 \log$ and 10\%: $4.19 \log$ ), S. marcescens CIP 103716 (80\%: 3.39 log, 50\%: $4.38 \mathrm{log}, 10 \%: 2.36 \mathrm{log}$ ) and ESBL-producing $K$. pneumoniae CTXM15 (80\%: $4.32 \mathrm{log}$ ), although a $\geq 3 \log$ decrease in CFU counts was still achieved.

\subsection{CHX-BZK Interaction and Final Formulation Validation}

According to preliminary assays on the CHX $0.2 \%$ - BZK 0.5\% combination, demonstrating bactericidal activity at a dilution of $0.5 \%$ and loss of activity at a dilution of $0.1 \%$ (data not shown), the concentrations tested for the interaction assessment ranged from $0.1 \%$ to $0.6 \%(\mathrm{v} / \mathrm{v})$. The mean logarithmic reductions in CFU count achieved against $S$. aureus CIP 4.83 and P. aeruginosa CIP 103467 after a 5 -min contact are presented in Table 4.

The bactericidal activity of the CHX-BZK combination was higher than that of each active substance tested separately, confirming a positive interaction between the two antiseptic agents. The CHX-BZK combination showed bactericidal activity at concentrations of $0.2 \%$ against $S$. aureus and $0.5 \%$ against $P$. aeruginosa, whereas these concentrations were inactive when the component antiseptic agents were tested individually (Table 4).

The licensed antiseptic solution Dermobacter ${ }^{\circledast}$ manifested a bactericidal activity similar to that of the $\mathrm{CHX}-\mathrm{BZK}$ solution, indicating no negative interaction with the excipients present in the formulation.

\section{Discussion}

The current requirement to establish that active substances or products under development, whatever their specific areas of application, have bactericidal activity is EN 1040 [5], a phase 1quantitative suspension test. However, this phase 1 test is insufficient to claim bactericidal activity in any particular clinical indication. To establish that a product has bactericidal activity under the practical conditions representative of its intended use, phase 2, step 1 quantitative 
Table 3. Bactericidal activity of Dermobacter ${ }^{\circledR}$ under "dirty" conditions according to the standard EN 13727 (contact time: $1 \mathrm{~min}$ ).

\begin{tabular}{llll}
\hline & \multicolumn{3}{c}{ Concentration (v/v) } \\
\cline { 2 - 4 } & $97 \% / 80 \%$ & $50 \%$ & $10 \%$ \\
\hline Mandatory strains & & & \\
Staphylococcus aureus CIP 4.83 & & \\
Pseudomonas aeruginosa CIP 103467 & & \\
Escherichia coli CIP 54.117 \\
Enterococcus hirae CIP 58.55 \\
Additional Gram + strains \\
Staphylococcus epidermidis CIP 6821 \\
Streptococcus pyogenes CIP $5641 \mathrm{~T}$ \\
Streptococcus agalactiae CIP 106884 \\
Corynebacterium amycolatum CIP $103452 \mathrm{~T}$ \\
Propionibacterium acnes CIP $53117 \mathrm{~T}$ \\
Clostridium perfringens CIP 103409 \\
Finegoldia magna CIP 103666 \\
Gardnerella vaginalis CIP $7074 \mathrm{~T}$
\end{tabular}

Additional Gram + strains with acquired antibiotic resistance

Staphylococcus aureus MRSA ATCC 33591

Staphylococcus aureus VISA CIP 106757

Enterococcus faecium VAN A CIP 107387

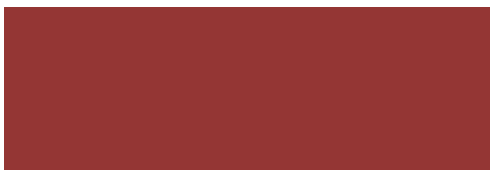

Additional Gram - strains

Klebsiella pneumonia CIP 8291

Enterobacter cloacae CIP $6058 \mathrm{~T}$

Serratia marcescens CIP 103716

Acinetobacter baumannii CIP 70.34T

Citrobacter freundii CIP $57.32 \mathrm{~T}$

Proteus mirabilis CIP 103181T

Bacteroides fragilis AIP 7716

Neisseria gonorrhoeae CIP 7918T

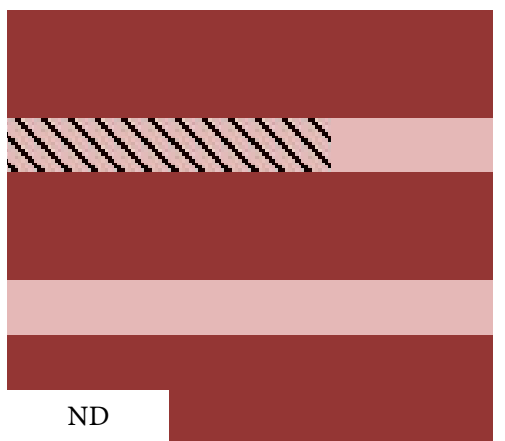

Additional Gram - strains with acquired antibiotic resistance

ESBL-producing Klebsiella pneumoniae CTXM15

Stenotrophomonas maltophilia tonoR

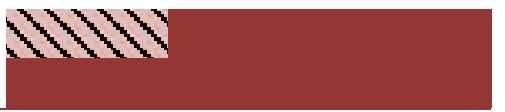

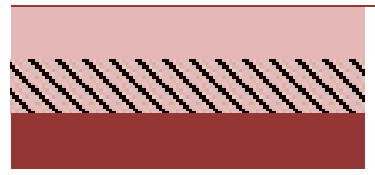

ND

$$
\begin{gathered}
\text { Log reduction }<3 \\
3 \leq \text { Log reduction }<5 \\
\text { Log reduction } \geq 5 \\
\text { Not determined }
\end{gathered}
$$

The theoretically inactive concentration tested was $1 \%$ or $0.1 \%$ (for the mandatory strains and for the additional strains, respectively; data not shown). Bactericidal activity is expressed as a logarithmic reduction at the tested concentrations $97 \% / 80 \%$ ( $97 \%$ for the mandatory strains, $80 \%$ for the additional strains with the exception of $S$. marcescens, $P$. mirabilis and $C$. perfringens for which both concentrations were tested), $50 \%$, and $10 \%$. 
Table 4. Bactericidal activity of chlorhexidine digluconate (CHX), benzalkonium chloride (BZK), the CHX-BZK combination* and the licensed antiseptic Dermobacter ${ }^{\circledast}$ against $S$. aureus CIP 4.83 and P. aeruginosa CIP 103467 according to the standard EN 1040.

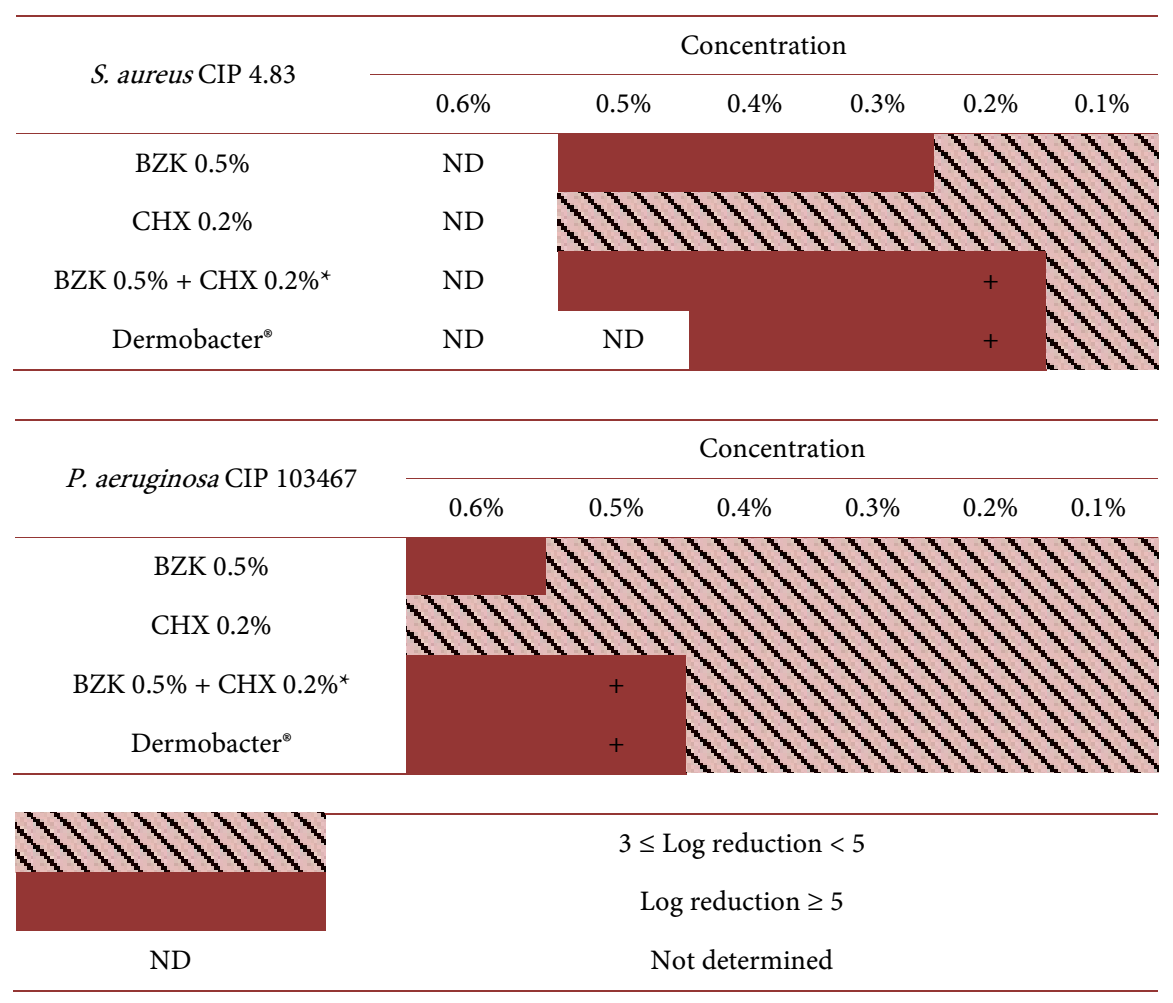

+: concentrations demonstrating a positive interaction between BZK and CHX.

${ }^{*}$ BZK $0.5 \%+$ CHX $0.2 \%$ corresponding to the same combination of active substances as in Dermobacter ${ }^{\circledast}$, without the excipients found in this commercial preparation.

suspension tests are required, such as those stipulated in the standard EN 13727, applicable to antiseptic products intended for hygienic or surgical hand rubs and washes [6].

Both these standards specify the use of mandatory bacterial strains including Gram-positive cocci belonging to the most important groups ( $S$. aureus and $E$. hirae), and the main representatives of Gram-negative bacilli (E. coli among the Enterobacteriaceae and $P$. aeruginosa). However, many other bacteria may be involved in infections and consequently need to be controlled by the use of antiseptics. For this reason, extended assays of bactericidal activity were performed on a licensed antiseptic (Dermobacter ${ }^{\circledast}$, containing the active substances CHX $0.2 \%$ and $\mathrm{BZK} 0.5 \%$ ) to validate its bactericidal activity against the four mandatory strains stipulated by the relevant European standards, in comparison to that manifested against a further 21 bacterial pathogens implicated in various mucocutaneous infections, some of them presenting acquired resistance to antibiotics.

First, the basic spectrum of bactericidal activity of the antiseptic was determined against a wide range of bacterial strains after 1 min of contact (at $20^{\circ} \mathrm{C}$ ) versus $5 \mathrm{~min}$ as recommended in the EN 1040 standard [5]. Under these defined conditions, the acceptance criterion for a log reduction in CFU count $\geq 5$ was 
met for the two mandatory strains and for $22 / 23$ of the additional strains tested, whatever the concentrations used for the assays. The exception was Proteus mirabilis, against which only a $\geq 3$ to $<5 \log$ reduction in CFU counts was observed at the highest concentrations ( $80 \%$ and $50 \%$ ).

In a second step, assays were performed under more drastic conditions, i.e. with dilution in hard water and in the presence of interfering substances corresponding to "dirty" conditions as defined in EN 13727 [6]. These assays similarly employed a contact time with the antiseptic of $1 \mathrm{~min}$ at $20^{\circ} \mathrm{C}$, and evaluated bactericidal activity against the four mandatory strains stipulated in EN 13727 and 21 additional strains. In this standard, a $5 \log$ reduction and a $3 \log$ reduction are required for hygienic hand-rub (highest tested concentrations: 97\%/80\%) and hand-wash (highest tested concentration: 50\%), respectively. The bactericidal activity of Dermobacter ${ }^{\otimes}$ under these conditions was maintained against the majority of the bacterial strains tested. However, no bactericidal activity against Proteus mirabilis was noted whatever the antiseptic concentration tested, and a decrease in the log reduction in CFU counts (from $\geq 5$ to $\geq 3$ under basic conditions to $<5$ ) against $C$. perfringens was detected at all antiseptic concentrations tested. Against $S$. marcescens, the antiseptic exhibited a $\geq 3$ to $<5 \log$ reduction in CFU counts at concentrations of $80 \%$ and $50 \%$, while the $10 \%$ concentration was inactive (log reduction $<3$ ). Against ESBL-producing $K$. pneumoniae CTXM15, the log reduction was decreased to $\geq 3$ to $<5$ at the $80 \%$ concentration but a $\geq 5 \log$ reduction was preserved at the $50 \%$ and $10 \%$ concentrations. Such a decrease in bactericidal activity at high concentrations has already been described with other antiseptics. In particular, the presence of certain excipients, particularly detergents, has been postulated to effect bactericidal activity depending on their concentrations [15] [20]. The coco alkyl dimethyl betaines contained in Dermobacter ${ }^{\otimes}$ have known detergent properties that may affect the bactericidal efficacy of this product against certain specific bacterial strains.

Comparison of the results obtained according to the two relevant European standards leads to the conclusion that 1) assays in which hard water is used to prepare dilutions of the antiseptic and that are conducted under "dirty" conditions are more discriminating than phase 1 tests to validate the intended use of a licensed antiseptic, 2) the bactericidal activity of the antiseptic evaluated was preserved in the presence of interfering substances (including minerals, proteins and erythrocytes), against the mandatory strains stipulated in the standards, as well as against most of the additional strains tested, with nevertheless some exceptions. As antiseptics are used in routine medical practice on both "clean" and "dirty" skin wounds, and the presence of whole blood or white cells is known to decrease their bactericidal activity, it is worth questioning why antiseptics intended for wound cleansing are still not required to meet standard criteria with interfering substances present. The draft revised version of EN 14485 currently under review does not envisage the use of "dirty" conditions for testing antiseptics. Consistently, in the very recently edited chapter of the European Pharmacopoeia (2017 edition) concerning determination of the bactericidal, fungicidal 
or yeasticidal activity of antiseptic medicinal products, the protocol described involves the use of only interfering substances corresponding to "clean" conditions and a contact temperature of $33^{\circ} \mathrm{C}$ [21]. Neither dilution of the product in hard water nor the use of "dirty" conditions is required. Moreover, the recommended contact time ( $5 \mathrm{~min}$ for bacteria and $15 \mathrm{~min}$ for fungi) does not appear to match the actual conditions of use.

Dermobacter ${ }^{\oplus}$ manifested a lower bactericidal activity against $P$. mirabilis than against the other bacterial strains tested, detected by the assay performed according to EN 1040 and confirmed by the assay conducted as specified in EN 13727 protocol. This finding was not surprising, given that $P$. mirabilis is considered as one of the species least susceptible to chlorhexidine among Gram-negative bacteria [22]. For three strains (two other Enterobacteriaceae and C. perfringens), a log reduction $<5$ but $\geq 3$ was noted in the EN 13727 assay, emphasizing that bactericidal activity persists and may be improved by a longer contact time. This is also suggested by the 5 -fold log reduction in $P$. mirabilis counts reached after a 5-min contact time under the conditions stipulated in EN 1040 [5] (result not shown). It is worth noting that under "dirty" conditions, the bactericidal activity of Dermobacter ${ }^{\circledast}$ against the five tested strains with acquired antibiotic resistance mechanisms-and chosen because of their potential cross-resistance to antiseptics-was maintained at all dilutions, except against ESBL-producing $K$. pneumoniae CTXM15 at the highest concentration (log reduction in CFU counts $\geq 3$ but $<5)$. Nevertheless, the issue of cross-resistance between antibiotics and antiseptics remains a matter of debate and controversy [23].

Finally, a previous study already underlined the value of adapting EN 13727 conditions to validate antiseptics intended for cutaneous and mucosal uses [15]. In that study, the bactericidal activity of the licensed antiseptic Dermobacter ${ }^{\circledast}$ (containing $0.2 \% \mathrm{CHX}$ and $0.5 \% \mathrm{BZK}$ ) against the mandatory strains was fully preserved under "dirty" conditions (log reduction $>5$ ) unlike that of the other tested products containing respectively povidone-iodine (PVI) and a combination of hexamidine, chlorhexidine and chlorocresol.

The results of our study confirm the choice of the mandatory strains stipulated in EN 1040 and EN 13727 to validate an antiseptic formulation. However, adding certain other strains to those currently specified in the standard, such as those suggested here (P. mirabilis, C. perfringens and S. marcescens) could be of interest, depending on the intended clinical use of the antiseptic tested, although further experiments with other antiseptic agents would be needed to confirm their relevance. Antibiotic-resistant strains could also be added, even though in this study these strains did not appear to be less susceptible than the other bacteria tested.

The interactions between CHX and BZK and the impact of the excipients of the licensed antiseptic tested were also investigated in our study. With respect to bactericidal activity against $S$. aureus, the active concentration of the $0.2 \% \mathrm{CHX}$ - $0.5 \%$ BZK combination was reduced by a third compared with those determined for CHX or BZK when tested separately. This positive interaction could 
result from the known activity of the two agents on the cell wall of Gram-positive bacteria wall, enhancing disruption of bacterial membrane before interaction with intracellular proteins [24] [25] [26] [27]. Against $P$. aeruginosa, the bactericidal activity determined for CHX and BZK when tested individually was maintained when these compounds were assayed in combination. Our results therefore confirm that these two cationic agents are compatible, have no negative interactions, and may even interact positively against some microorganisms.

The licensed antiseptic tested exhibited a bactericidal activity similar to that of the combination of its two active substances alone (CHX 0.2\% - BZK 0.5\%). This result emphasizes that the excipients contained in the marketed formulation did not impair the bactericidal efficacy of these antiseptic agents against the tested strains. This is in line with the report published by Cowley et al. (2015), suggesting that many formulated microbicides may even exhibit greater antibacterial potency than their unformulated active substances [28].

Resistance to antiseptics, including chlorhexidine [1] [22], but also QACs [29] [30], is becoming a challenge, particularly in the presence of interfering substances. This phenomenon leads to the selection of microbicide-resistant bacteria. In this context, the results mentioned above stress the need for further investigations on antiseptic combinations. It may be hypothesized that the combination of active substances that act on similar or different targets on bacterial cells could be an interesting approach to limit or counteract the development of resistance, notably by reducing their respective active concentrations and/or enlarging their spectra of bactericidal activity. Benefits of such combinations have been reported, for instance, with respect to hydroalcoholic solutions of CHX and PVI [31] [32] [33]. Similarly, BZK has been shown to be a key factor for the preservation of bactericidal activity of a CHX $0.2 \%$ - BZK $0.5 \%$ formulation in the presence of interfering substances, the activity of $\mathrm{CHX} 0.2 \%$ alone being inhibited by $0.3 \%$ bovine albumin, whereas the combination CHX $0.2 \%$ - BZK 0.5\% remained bactericidal [14].

In conclusion, the licensed antiseptic tested (Dermobacter ${ }^{\oplus}$; CHX 0.2\% - BZK $0.5 \%$ ) exhibited a broad spectrum of bactericidal activity in vitro, including activity against strains with acquired antibiotic resistance. Its bactericidal activity against the majority of the strains tested was unchanged under "dirty" conditions and may be expected to remain unchanged in vivo, within its range of indications concerning the prevention and treatment of cutaneous and mucosal infections.

The results obtained with this licensed antiseptic support EN 14885 recommendations regarding phase 2 standards for newly marketed products and indicate that the mandatory strains stipulated in the standards EN 1040 [5] and EN 13727 [6] are representative to assess the bactericidal activity of antiseptic. The 1 min contact time and the presence of interfering substances appear to be the most appropriate testing conditions for mucocutaneous antiseptics. However, considering the decrease in the bactericidal activity of Dermobacter ${ }^{\circledast}$ against a 
few strains observed under "dirty conditions", attention should be paid by manufacturers to the relevance of adding certain other strains to those stipulated in standards, according to the intended clinical use of the antiseptic to be tested, in order to avoid potential misuse.

\section{Acknowledgements}

This project was supported by Laboratoire Innotech International.

\section{Conflicts of Interest}

The authors declare no conflicts of interest regarding the publication of this paper.

\section{References}

[1] Horner, C., Mawer, D. and Wilcox, M. (2012) Reduced Susceptibility to Chlorhexidine in Staphylococci: Is It Increasing and Does It Matter? Journal of Antimicrobial Chemotherapy, 67, 2547-2559. https://doi.org/10.1093/jac/dks284

[2] Messager, S., Goddard, P.A., Dettmar, P.W. and Maillard, J.Y. (2001) Determination of the Antibacterial Efficacy of Several Antiseptics Tested on Skin by an “EX-Vivo” Test. Journal of Medical Microbiology, 50, 284-292. https://doi.org/10.1099/0022-1317-50-3-284

[3] Crémieux, A., Fleurette, J., Fourtillan, J.B., Joly, B. and Soussy, C.J. (1982) Les Antiseptiques. Bases Microbiologiques de leur Utilisation, Ed Sarget, Paris, 71.

[4] AFNOR (2015) NF EN 14885-Chemical Disinfectants and Antiseptics-Application of European Standards for Chemical Disinfectants and Antiseptics. AFNOR, La Plaine Saint-Denis.

[5] AFNOR (2006) NF EN 1040-Chemical Disinfectants and Antiseptics-Quantitative Suspension Test for the Evaluation of Basic Bactericidal Activity of Chemical Disinfectants and Antiseptics-Test method and Requirements (Phase 1). AFNOR, La Plaine Saint-Denis.

[6] AFNOR (2015). NF EN 13727 + A2-Chemical Disinfectants and Antiseptics-Quantitative Suspension test for the Evaluation of Bactericidal Activity in the Medical Area-Test Method and Requirements (Phase 2, Step 1). AFNOR, La Plaine Saint-Denis.

[7] Crémieux, A., Chevalier, J. and Dauriac, H. (1975) Évolution de la Concentration Bactéricide de Désinfectants en fonction de la Présence de Diverses Substances Interférentes. Revue de l'Institut Pasteur de Lyon, 8, 187-197.

[8] Hood, R., Shermock, K.M. and Emerman, C. (2004) A Prospective, Randomized Pilot Evaluation of Topical Triple Antibiotic versus Mupirocin for the Prevention of Uncomplicated Soft Tissue Wound Infection. American Journal of Emergency Medicine, 22, 1-3. https://doi.org/10.1016/j.ajem.2003.09.009

[9] Bonomo, R.A., Van Zile, P.S., Li, Q., Shermock, K.M., McCormick, W.G. and Kohut, B. (2007) Topical Triple-Antibiotic Ointment as a Novel Therapeutic Choice in Wound Management and Infection Prevention: A Practical Perspective. Expert Review of Anti-Infective Therapy, 5, 773-782. https://doi.org/10.1586/14787210.5.5.773

[10] Gélinas, P. and Goulet, J. (1983) Neutralization of the Activity of Eight Disinfec- 
tants by Organic Matters. The Journal of Applied Bacteriology, 54, 243-247. https://doi.org/10.1111/j.1365-2672.1983.tb02613.x

[11] Chantefort, A. and Druilles, J. (1984) Activité Bactéricide de Quelques Désinfectants en Présence ou Non de Substances Interférentes Protéiques. Pathologie Biologie, 32, 615-618.

[12] Guiraud-Dauriac, H. and Crémieux, A. (1984) Inactivation par les Protéines et les Ions Calcium des Désinfectants en Fonction de leur Nature Chimique et des Espèces Bactériennes. Pathologie Biologie, 32, 611-614.

[13] Crémieux, A., Bonnaveiro, N. and Chevalier, J. (1987) Intérêt d'un Exsudat Standard dans l'Etude de l'Activité In Vitro des Antiseptiques. Pathologie Biologie, $35,887-890$.

[14] Cabotin, P.P. and Bertrand, J.C. (2004) Étude Comparative de l'Activité Bactéricide de Deux Antiseptiques Cutanés en Solution Aqueuse, en Présence de Substances Interférentes. Nouvelles Dermatologiques, 23, 236-238.

[15] Salvatico, S., Feuillolay, C., Mas, Y., Verrière, F. and Roques, C. (2015) Bactericidal Activity of 3 Cutaneous/Mucosal Antiseptic Solutions in the Presence of Interfering Substances: Improvement of the NF EN 13727 European Standard? Médecine et Maladies Infectieuses, 45, 89-94. https://doi.org/10.1016/j.medmal.2015.01.006

[16] Fleurette, J., Freney, J. and Reverdy, M.E. (1995) Antisepsie et Désinfection. Ed Eska, Paris.

[17] Bereket, W., Hemalatha, K., Getenet, B., Wondwossen, T., Solomon, A., Zeynudin, A. and Kannan, S. (2012) Update on Bacterial Nosocomial Infections. European Review for Medical and Pharmacological Sciences, 16, 1039-1044.

[18] Teresa, C.H., Andrus, M. and Dudeck, M.A. (2008) CDC/NHSN Surveillance Definition of Health Care-Associated Infection and Criteria for Specific Types of Infections in the Acute Care Setting. American Journal of Infection Control, 36, 301-308.

[19] FDA, Department of Health and Human Services (2015) Safety and Effectiveness of Health Care Antiseptics; Topical Antimicrobial Drug Products for Over-the-Counter Human Use; Proposed Amendment of the Tentative Final Monograph; Reopening of Administrative Record; Proposed Rule.

[20] Chevalier, J., Corre, J. and Crémieux, A. (1995) Evaluation of Synergistic Effects of Three Bactericidal Agents Associated in an Antiseptic Formulation. Pharmaceutica Acta Helvetiae, 70, 155-159. https://doi.org/10.1016/0031-6865(95)00015-2

[21] European Pharmacopoeia (2017) Determination of Bactericidal, Fungicidal or Yeasticidal Activity of Antiseptic Medicinal Products. Version 9.2, Chapter 5.1.11.

[22] Stickler, D.J. (2002) Susceptibility of Antibiotic-Resistant Gram-Negative Bacteria to Biocides: A Perspective from the Study of Catheter Biofilms. Journal of Applied Microbiology, 92, 163S-170S.

[23] Kampf, G. (2016) Acquired Resistance to Chlorhexidine-Is It Time to Establish an “Antiseptic Stewardship” Initiative? Journal of Hospital Infection, 94, 213-227. https://doi.org/10.1016/j.jhin.2016.08.018

[24] Lim, K.S. and Kam, P.C.A. (2008) Chlorhexidine-Pharmacology and Clinical Applications. Anaesthesia and Intensive Care, 36, 502-512.

[25] Kodedova, M., Sigler, K., Lemire, B.D. and Gaskova, D. (2011) Fluorescence Method for Determining the Mechanism and Speed of Action of Surface-Active Drugs on Yeast Cells. BioTechniques, 50, 58-63. https://doi.org/10.2144/000113568

[26] Cheung, H.Y., Wong, M.M.K., Cheung, S.H., Liang, L.Y., Lam, Y.W. and Chiu, S.K. (2012) Differential Actions of Chlorhexidine on the Cell Wall of Bacillus subtilis 
and E. coli. PLoS ONE, 7, e36659. https://doi.org/10.1371/journal.pone.0036659

[27] Vijayakumar, R., Kannan, V.V., Sandle, T. and Manoharan, C. (2013) In Vitro Antifungal Efficacy of Biguanides and Quaternary Ammonium Compounds against Clean Room Fungal Isolates. Journal of Pharmaceutical Science and Technology, 66, 236-242.

[28] Cowley, N.L., Forbes, S., Amézquita, A., McClure, P., Humphreys, G.J. and McBain, A.J. (2015) Effects of Formulation on Microbicide Potency and Mitigation of the Development of Bacterial Insusceptibility. Applied and Environmental Microbiology, 81, 7330-7338. https://doi.org/10.1128/AEM.01985-15

[29] Babaei, M., Sulong, A., Hamat, R., Nordin, S. and Neela, V. (2015) Extremely High Prevalence of Antiseptic Resistant Quaternary Ammonium Compound E Gene among Clinical Isolates of Multiple Drug Resistant Acinetobacter baumannii in Malaysia. Annals of Clinical Microbiology and Antimicrobials, 14, 11. https://doi.org/10.1186/s12941-015-0071-7

[30] Taheri, N., Ardebili, A., Amouzandeh-Nobaveh, A. and Ghaznavi-Rad, E. (2016) Frequency of Antiseptic Resistance among Staphylococcus aureus and Coagulase-Negative Staphylococci Isolated from a University Hospital in Central Iran. Oman Medical Journal, 31, 426-432. https://doi.org/10.5001/omj.2016.86

[31] Parienti, J.J., du Cheyron, D., Ramakers, M., Malbruny, B., Leclercq, R., Le Coutour, X., Charbonneau, P. and Members of the NACRE Study Group (2004) Alcoholic Povidone-Iodine to Prevent Central Venous Catheter Colonization: A Randomized Unit-Crossover Study. Critical Care Medicine, 32, 708-713. https://doi.org/10.1097/01.CCM.0000115265.05604.7B

[32] Adams, D., Quayum, M., Worthington, T., Lambert, P. and Elliott, T. (2005) Evaluation of a $2 \%$ Chlorhexidine Gluconate in 70\% Isopropyl Alcohol Skin Disinfectant. Journal of Hospital Infection, 61, 287-290. https://doi.org/10.1016/j.jhin.2005.05.015

[33] Chiang, S.R., Jung, F., Tang, H.J., Chen, C.H., Chen, C.C., Chou, H.Y. and Chuang, Y.C. (2017) Desiccation and Ethanol Resistances of Multidrug Resistant Acinetobacter baumannii Embedded in Biofilm: The Favorable Antiseptic Efficacy of Combination Chlorhexidine Gluconate and Ethanol. Journal of Microbiology, Immunology and Infection. 